\title{
Active Head Motion Compensation of TMS Robotic System Using Neuro- Fuzzy Estimation
}

\author{
W.N. Wan Zakaria, Razali Tomari and Rafidah Ngadengon \\ Advanced Mechatronic Research Group (ADMIRE), Faculty of Electrical \& Electronic Engineering, Universiti Tun Hussein Onn Malaysia, \\ 86400 Parit Raja, Batu Pahat, Johor, Malaysia.
}

\begin{abstract}
Transcranial Magnetic Stimulation (TMS) allows neuroscientist to study human brain behaviour and also become an important technique for changing the activity of brain neurons and the functions they sub serve. However, conventional manual procedure and robotized TMS are currently unable to precisely position the TMS coil because of unconstrained subject's head movement and excessive contact force between the coil and subject's head. This paper addressed this challenge by proposing an adaptive neuro-fuzzy force control to enable low contact force with a moving target surface. A learning and adaption mechanism is included in the control scheme to improve position disturbance estimation. The results show the ability of the proposed force control scheme to compensate subject's head motions while maintaining desired contact force, thus allowing for more accurate and repeatable TMS procedures.
\end{abstract}

\section{Introduction}

Recent developments in the neuroscience area have heightened the need of robotized Transcranial Magnetic Stimulation (TMS) system to study human brain behaviour. In the TMS procedure, an electromagnetic coil is placed over the head, and a large but brief current pulse is produced and passed through the scalp [1]. The changing magnetic field which this generates induces a current in the conductive tissue of the head including the underlying cerebral cortex.

The cortical activity produced by TMS is critically dependent on coil location and orientation. Currently, the coil is positioned manually, which can be a problem for precise clinical evaluation and experiments of the TMS technique.

Although the TMS technique is very useful, it is not yet widely accepted because of the observed inconsistency of efficiency between subjects. TMS coils produce very dense and defined fields, which mean that a small movement of the coil position leads to a substantial change of the electromagnetic field delivered to a target nerve. It is due to the difficulty to indicate correct positioning with the current available stimulation systems which leads to poor repeatability [2][3].

This leads to applications of robotics in TMS to position the coil for efficient and reliable TMS procedures. Robot assistance in TMS procedure has been reported by a number of researchers [2-6]. These existing TMS robotic systems, which aim at replacing the hand and the arm of neurologist, are particularly focused on image-guided by means of navigation system to position the coil on subject's head.

Despite its safety and efficacy, current researchers are unable to address the force control as an important feature of the TMS procedure. Most of the research to date has tended to focus on image-guided and navigation system and neglected the study on contact force between the coil and the subject's head which is crucial. Excessive contact force between the coil and unconstrained subject's head may harm the subject.

During conventional hand-held procedure test, the following issues were observed and can be considered as challenges to the force-controlled TMS robot system implementation;

1. The applied force exerted by the operator along normal direction to the subject's head surface was found ranging from $6 \mathrm{~N}$ to $10 \mathrm{~N}$ in which the operator keeps the coil in close contact to the hot-spot position.

2. The subject's head geometrical, inertial and structural stiffness properties are totally unknown and vary from one subject to other subjects.

3. During the treatment, even the subject is in relax and conscious condition there is a higher possibility of involuntary head movement due to tremor, muscle contraction or relaxation involving to-and-from movement. 


\section{Human head-neck motion estimation}

Human head-neck movement is extremely complex and is not completely understood. There is a considerable amount of literature published on this topic, however it is still impossible to model the head-neck system totally because of several factors namely; head-neck sheer complexity and lack of quantitative data on material properties, geometries and boundary conditions of individual tissues [7-11]. The outcome suggests that there is tremendous variation in the types of biomechanical models to approximate the human head-neck system [12]. This is due to the consideration of task, for example, slow voluntary movement is differed than movement resulting from an impact to the head or during walking. In addition, researchers also suggest those horizontal and vertical movement kinematics is coupled as the same muscles are involved in both types of movement [7] [12]. Typically, the movement is assumed to be a straight path with a single peak and bell-shaped velocity profile [13-15].

The problem is simplified from a task specification point of view in which force control is executed along the normal direction to the subject's head surface. Thus, it is necessary to establish the translational movement of the unconstrained head in relax and seated condition along this direction. A linear relationship between rotational and translational movement was established as described in following section.

\subsection{Ellipsoidal shaped head geometry}

An ellipsoidal shape was chosen to define the head geometry as it exhibits elevation as well as azimuth dependence [16]. To express the head geometry analytically, consider the head pitch movement $\left(\theta_{p}\right)$ around the rotation axis which is the centre of the ellipsoid as shown in Figure 1.

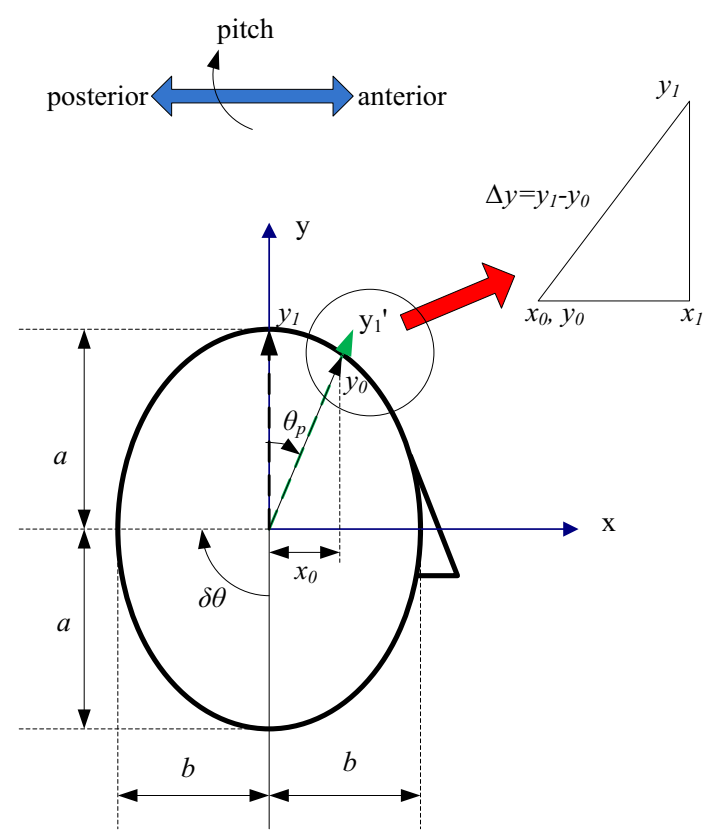

Figure 1. Ellipsoidal shaped head geometry $\theta_{p}$ : head pitch movement, $a$ : distance from a centre rotation, $b$ : half head width

As the head rotates about $\theta_{p}$ from start position $y_{l}$ to final position $y_{0}$, the translational distance can be expressed as $\Delta y=y_{1}-y_{0}$. The following heuristic procedure is defined and yields a close approximation to determine $\Delta y$;

Find the point $y_{0}$,

$$
y_{0}=\frac{x_{0}}{\tan \theta_{p}}
$$

Find $x_{0}$ by using following ellipsoid equation;

$$
\frac{x_{0}^{2}}{a^{2}}+\frac{y_{0}^{2}}{b^{2}}=1
$$

Define the $y_{0}$ and gradient $m$ as both $y_{1}$ and $y_{0}$ positions lie on the same straight line,

$$
m=\frac{x_{0}}{y_{0}}=\frac{x_{1}}{y_{1}}=\frac{1}{\tan \theta_{p}}
$$

Combining equations (1), (2) And (3), thus $x_{0}$

$$
x_{0}=\sqrt{\frac{a^{2} b^{2}}{\left(\frac{a}{\tan \theta_{p}}\right)^{2}+b^{2}}}
$$

where, $a$ is a distance from centre of rotation axis (approximately $30 \mathrm{~mm}$ inferior to the interaural axis [11] to the $y_{1}$ point and $b$ is the half of human head width). Based on [8][11][17] works, $a, b$ and $\theta_{p}$ can be defined as $160 \mathrm{~mm}, 95 \mathrm{~mm}$ and $10^{\circ}$ respectively.

For further analysis, the head-neck [12] is related as following equation;

$$
\frac{d x}{d t .}=r \cdot \frac{d \theta}{d t .} \approx \vartheta=r . \omega
$$

where $r,(x, \theta)$ and $(v, \omega)$ are the vector directed from the axis of rotation, translational and angular position and translational and angular velocity respectively. To guide this analysis, the head movement parameters were estimated by considering various research studies. Ferman et al. [8] suggest that the subject head oscillate about $0.25 \mathrm{rad} / \mathrm{s}$ to $1.05 \mathrm{rad} / \mathrm{s}$ (amplitude about 10 degrees) in the horizontal, vertical or torsional plane during seated position while fixating target at optical infinity. This corresponds to $40-160 \mathrm{~mm} / \mathrm{s}$ translational velocities. This is almost consistent with the findings of [18] that investigated the amplitude of head rotation for the subject seated stationary in defined 'relax' and 'still' position with the head in a resting and facing forward. Their studies found the approximately similar oscillations of $0.5 \mathrm{rad} / \mathrm{s}$ to $1.5 \mathrm{rad} / \mathrm{s}$ in both conditions. These calculation parameters can be considered as motion disturbances to the force controller and will be used in following experimental programme.

\section{Force Control Scheme}

Figure 2 illustrates the block diagram of the force control architecture based on [19-22] as implemented in the TMS robotic system. Both internal position and external force control loops are performed in Cartesian coordinates. 


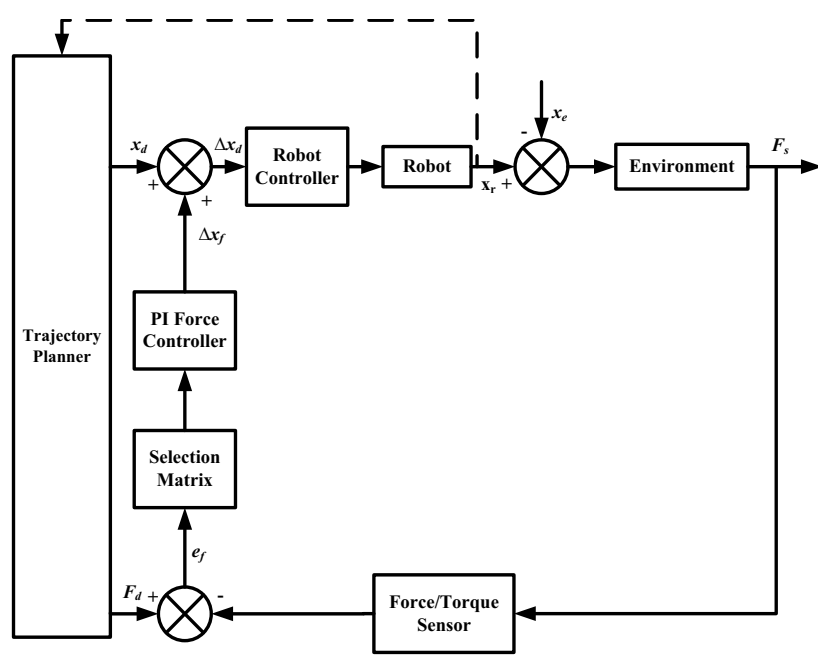

Figure 2. The external force control scheme

The key idea of this control strategy is that the force control loop generates the corrective position data from the force error, $e_{f}\left(t_{k}\right)$ through the Proportional-Integral (PI) force controller. The initial contact position $x_{d}\left(\mathrm{t}_{\mathrm{k}-1}\right)$ is then modified by the force controller output $\Delta x_{f}\left(t_{k}\right)$. The resultant position demand $\Delta x_{d}\left(t_{k}\right)$ is forwarded to robot controller to determine the next desired position of the robot at every path update interval.

Prior to programming the robot to perform the TMS operation tasks, the issue relating to force control stability was first addressed. The implementation of a conventional PI force controller in TMS robotic system was found to be difficult. The preliminary test result suggests that unknown or uncertain environment stiffness and positional disturbance would drive the system into undesired behaviour. Thus, an intelligent approach is proposed to overcome these identified problems as discussed in the following section.

\subsection{Adaptive Neuro-Fuzzy Force Controller based on MRAC}

The Adaptive Neuro-Fuzzy Force Controller (ANFFC) structure is similar to that of a classical model reference adaptive control (MRAC) method as shown in Figure 3. The general idea of MRAC is to create a 'parameter adjustment' algorithm based on the model error $\varepsilon$ to change the system response to match a desired model. The output of the parameter adjustment algorithm $\delta$ approaches unity as the system response tends to desired response.

The ANFFC consists of three main components: a reference model describing the desired behaviour of the force control scheme, a main Neuro-Fuzzy force controller (NFFC) that determines the adjustment to the position control loop and Neuro-Fuzzy learning and adaption mechanism (NFLA) that modifies the NFFC output.

The implementation of ANFFC is to compensate for any unknown or significant change in environment parameters that may lead to overshoot and undershoot error responses. This method behaves in a similar way to a human does, as we do not apply every control rule for particular situation but moderately adjusts the existing
Neuro-Fuzzy Force Controller (NFFC) corresponding to the actual situation. The Neuro-Fuzzy [23-25] concept is applied to determine and generate the effective parameter adjustment algorithm.

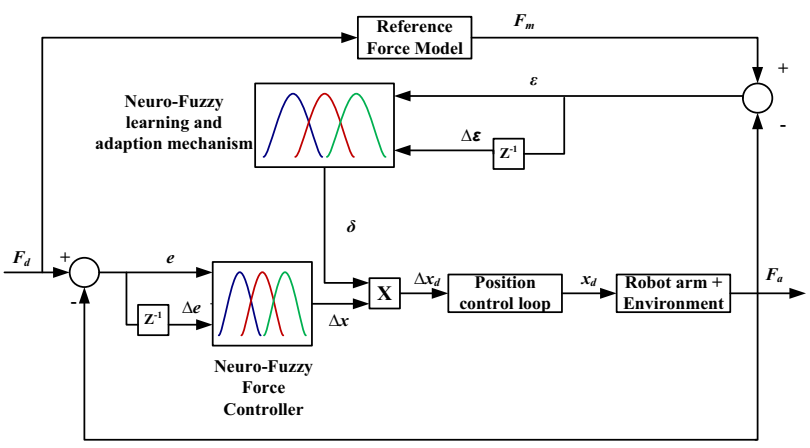

Figure 3. The Adaptive Neuro-Fuzzy Force Controller (ANFFC) structure

\subsubsection{Reference Force Model}

The reference model (equation 6) is chosen to generate the whole system desired behaviour in response to a force trajectory. In this research, the reference model is generated using the MATLAB ${ }^{\circledR}$ System Identification Toolbox $^{\mathrm{TM}}$ software. Prior to that, a step force response test was conducted to identify the input-output data set of desired behaviour of force control system. The desired behaviour criteria are $0.2 \mathrm{~s}$ rise time, overshoot of $10 \%$ with $5 \%$ steady-state error.

The input-output data was used to estimate a secondorder linear system parameters and the model output is validated by using model-output plot where the identified model accurately fit the measured data of $\sim 90 \%$.

$$
\frac{F_{m}}{F_{d}}=\frac{1}{s^{2}+7 s+100}
$$

where damping ratio, $\zeta$ and natural frequency, $\omega$ are 0.35 and 10 respectively.

\subsubsection{Design of Neuro-Fuzzy learning and adaption mechanism}

The purpose of the NFLA is to learn and adapt to any changes in the environmental parameters and subsequently modify the NFFC output accordingly so that the overall system response is as close as possible to the desired reference model. The NFLA inputs are the difference between the reference model output and the measured contact force $\varepsilon$ and the rate of error $\Delta \varepsilon$. Its output is the parameter of factor adjustment $\delta$ which is used to modify the output of NFFC. The method is very useful in compensating any disturbance caused by environment position (moving object) which is a key requirement for the TMS robotic system. The NeuroFuzzy approach was applied to evaluate the degree of influence of the undesired response, and consequently, the adjustment algorithm generates a larger factor adjustment parameter for the larger undesired oscillation and unexpected overshoot/undershoot error. Similarly a 
smaller value is generated if the measured force is closed to the desired response.

The rule-base is created as follows;

If $\varepsilon(\boldsymbol{k})$ is $\varepsilon_{i}$ and $\Delta \varepsilon(\boldsymbol{k})$ is $\Delta \varepsilon_{i}$, then output is $\boldsymbol{\delta} \boldsymbol{i}$ To begin with, the environment movement can be classified in terms of the sign of the model error $\varepsilon$ :

1) Negative if the object moves towards the Stäubli robot

2) Positive if the object moves away from the Stäubli robot

Similarly the force controller behaviour can be defined by the rate of error sign as summarized in Table 1.

Table 1. A rule-base parameter determination

\begin{tabular}{|c|c|c|c|}
\hline$\Delta \boldsymbol{\varepsilon}$ sign & $\boldsymbol{\varepsilon}$ sign & $\begin{array}{c}\text { Force } \\
\text { controller } \\
\text { response }\end{array}$ & Adjustment \\
\hline Zero & $\begin{array}{c}\text { Zero/Positive/ } \\
\text { Negative }\end{array}$ & Satisfactory rate & $\begin{array}{c}\text { No or small } \\
\text { adjustment }\end{array}$ \\
\hline Positive & Positive & Slow & $\begin{array}{c}\text { Large } \\
\text { adjustment }\end{array}$ \\
\cline { 2 - 4 } & Negative & Satisfactory rate & $\begin{array}{c}\text { Small } \\
\text { adjustment }\end{array}$ \\
\hline Negative & Positive & Satisfactory rate & $\begin{array}{c}\text { Small } \\
\text { adjustment }\end{array}$ \\
\cline { 2 - 4 } & Negative & Slow & adjustment \\
\hline
\end{tabular}

\section{Experimental Evaluation}

This section describes how the force controller is able to accommodate a dynamic environment which is not stationary; namely a sequence of ramp inputs with/without parameter adjustment algorithms. Figure 4 illustrates the schematic diagram of ANNFC test set up.

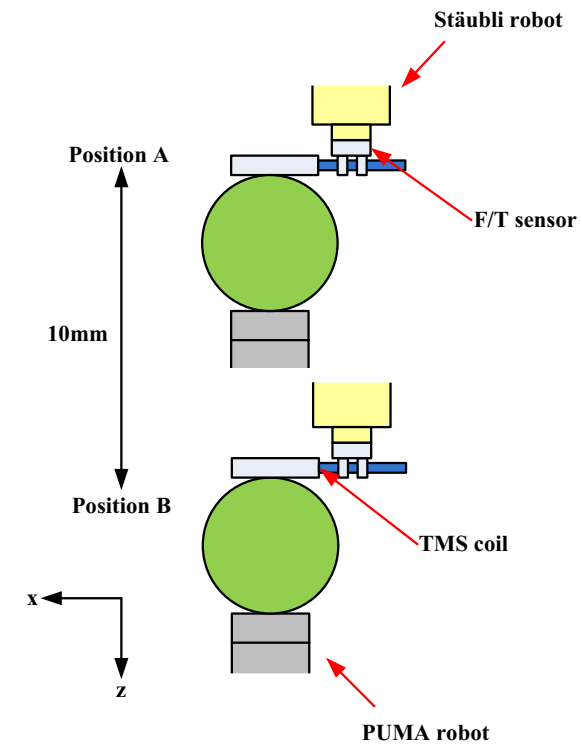

Figure 4. ANFFC experimental test set up

The Stäubli robot is first moved down at an approximate $10 \mathrm{~mm} / \mathrm{sec}$ until it makes contact with the ball. Once contact is made, the force control law is activated in which the robot was commanded to maintain $5 \mathrm{~N}$ contact force in the $z$ axis direction. Then, after approximately 7 seconds the PUMA robot is moved about $10 \mathrm{~mm}$ in towards direction (from position B to A) to the Stäubli robot with a speed approximately $2 \mathrm{~mm} / \mathrm{sec}$. Another PUMA robot movement (from position A to B) was applied for the same travel distance. Note that, neither environment stiffness nor its position is known to the robot controller.

\section{Results and Discussion}

Figure 5 shows the results of force and position (along $\mathrm{z}$ axis) during this test. There was a significant difference between the conventional PI and the ANFFC controllers. It is apparent from the data in Figure 5, that the conventional PI force controller drives the manipulator into an unstable limit cycle. The problem can be attributed to the rapid changes in movement (either robot or environment) that leads to impulsive forces on the system. The form of the signal shows the PI controller was trying to compensate the impulsive force, but failed to do so. In this condition, it is desirable if the Stäubli robot can moves at velocity equal to the environment speed.
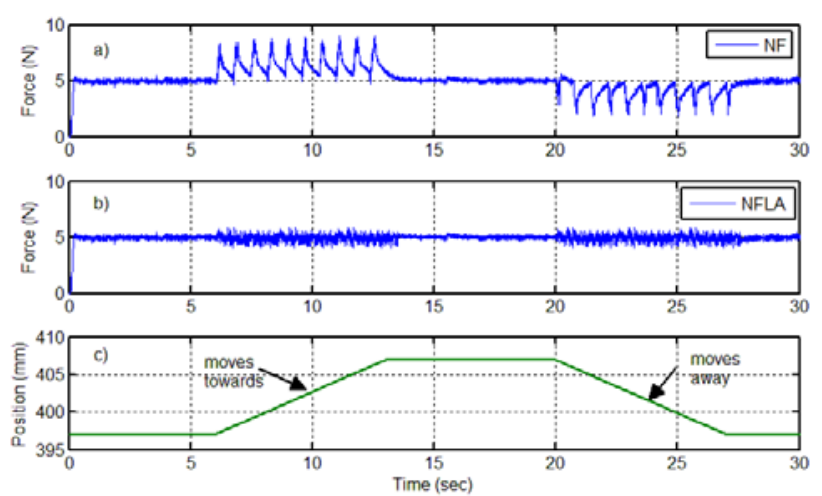

Figure 5. Test results of force response of (a) PI and (b) NFLA, and (c) Stäubli robot position

Consider the situation in which the environment moves towards the robot very rapidly, the large force error occurs before the robot controller can react to it due to small calculated incremental position. This results in the robot springing backward rapidly. Similar pattern is observed during away movement, if the environment movement speed is higher than resultant incremental position, the robot momentarily leaving contact with the environment. This inevitably results in repetitive bouncing action until the environment stop moving. Clearly, the dynamics of the force-controlled system are now considered to be extremely complex with many unknown independent factors. Thus the task of maintaining force response, as position changing with the conventional PI controller only is impossible.

\section{Conclusion}


The PI gain scheduling and Neuro-Fuzzy methods were successfully implemented in TMS robotic system and demonstrated improvement for the control system. Experimental results have shown the effectiveness of the proposed controller particularly for unknown/variable environment stiffness as well as in condition when the environment parameters and position are unknown and change substantially. In addition, the proposed method also employs a learning and adaptive mechanism to adjust a main loop Neuro-Fuzzy force controller online. The purpose of this mechanism is to learn the environment and adapt the controller accordingly. Adaption consists of comparing the model reference error and using the derivative of error as the input to the Neuro-Fuzzy algorithm. Any undesired response is immediately compensated with a suitable adjustment parameter according to the response behaviour.

\section{Acknowledgement}

This work was supported by Ministry of Education and Universiti Tun Hussein Onn Malaysia under Research Acculturation Grant Scheme Vot R032.

\section{References}

1. M. Hallett, 'Transcranial magnetic stimulation: A primer', Neuron, 55, (2), pp. 187-199. (2007)

2. L. Matthäus, A. Giese, D. Wertheimer, and A. Schweikard, 'Planning and analyzing robotized TMS using virtual reality', Studies in health technology and informatics, 119,pp. 373-378. (2006)

3. L. Matthäus, P. Trillenberg, and C. Bodensteiner, 'Robotized TMS for motion compensated navigated brain stimulation', International Journal of Computer Assisted Radiology and Surgery, 1, (SUPPL. 7), pp. 139-141. (2006)

4. M. Finke, T. Fadini, S. Kantelhardt, A. Giese, L. Matthaus, and A. Schweikard, 'Brain-Mapping using robotized TMS', International Conference of the IEEE Engineering in Medicine and Biology Society. Vancouver, CANADA, Aug 20-24. pp. 3929-3932. (2008)

5. J. Lancaster, S. Narayana, D. Wenzel, J. Luckemeyer, J. Roby, and P.Fox. 'Evaluation of an image-guided, robotically positioned transcranial magnetic stimulation system', Human Brain Mapping, 22, (4), pp. 329-340. (2004)

6. C. Lebosse, P. Renaud, B. Bayle, M. De Mathelin, O. Piccin, and $\mathrm{J}$ Foucher, 'A robotic system for automated image-guided Transcranial Magnetic Stimulation', IEEE/NIH Life Science Systems and Applications Workshop, LISA. Bethesda, MD, pp. 55-58. (2008)

7. BHannaford, W.S.Kim, S.H. Lee, and L Stark, 'Neurological control of head movements: Inverse modeling and electromyographic evidence', Mathematical Biosciences, 78, (2), pp. 159-178. (1986)

8. L. Ferman, H. Collewijn, T.C Jansen, and A. V. Van den Berg, 'Human gaze stability in the horizontal, vertical and torsional direction during voluntary head movements, evaluated with a three-dimensional scleral induction coil technique', Vision Research, 27, (5), pp. 811-828. (1987)

9. J. Winters, 'Biomechanical Modeling of the Human Head and Neck', in F.J.Richmond, B. W. P. a.(ed),
Control of Head Movement. Oxford University Press. (1988)

10. A. Hagemann, K. Rohr, H.S. Stiehl, U. Spetzger, and J.M. Gilsbach, 'Biomechanical modeling of the human head for physically based, nonrigid image registration', Medical Imaging, IEEE Transactions on, 18, (10), pp. 875-884. (1999)

11. S. T. Moore, E. Hirasaki, T. Raphan, and B. Cohen, 'Instantaneous rotation axes during active head movements', Journal of Vestibular Research, 15, (2), pp. 73-80. (2005)

12. B. W. Peterson, and F. J. Richmond, 'Control of head movement'. Oxford University Press, USA. (1988)

13. R. Plamondon, 'A kinematic theory of rapid human movements - Part I. Movement representation and generation', Biological Cybernetics, 72, (4), pp. 295307. (1995)

14. R. Plamondon, C. Feng, and A. Woch, 'A kinematic theory of rapid human movement. Part IV: A formal mathematical proof and new insights', Biological Cybernetics, 89, (2), pp. 126-138. (2003)

15. J. T. Cavanaugh, D. Goldvasser, C. A. McGibbon, and D. E. Krebs, 'Comparison of head- and bodyvelocity trajectories during locomotion among healthy and vestibulopathic subjects', Journal of Rehabilitation Research and Development, 42, (2), pp. 191-198. (2005)

16. R. O. Duda, C. Avendano, and V.R. Algazi, 'An adaptable ellipsoidal head model for the interaural time difference', IEEE International Conference onAcoustics, Speech, and Signal Processing, 15-19 Mar 1999. pp. 965-968 vol.2. (1999)

17. P. Stephen, 'Bodyspace: anthropometry, ergonomics, and the design of work'. Taylor \& Francis. (1996)

18. R. Chan, D. K. Rogers, and D. I. McCloskey, 'Postural stability of the head in response to slowly imposed, small elastic loads', Neuroscience Letters, 214, (2-3), pp. 205-207. (1996)

19. J. De Schutter, 'A study of active compliant motion control methods for rigid manipulators based on a generic scheme', Robotics and Automation. Proceedings. 1987 IEEE International Conference on. Mar 1987. pp. 1060-1065. (1987)

20. J. De Schutter, and H. Van Brussel, 'Compliant Robot Motion I. A Formalism for specifying compliant motion tasks', International Journal of Robotics Research, 7, (4), pp. 3-17. (1988)

21. J. De Schutter, and H. Van Brussel, 'Compliant Robot Motion II. A Control Approach Based On External Control Loops', International Journal of Robotics Research, 7, (4), pp. 18-33. (1988)

22. E. Degoulange, and P. Dauchez, 'External force control of an industrial PUMA 560 robot', Journal of Robotic Systems, 11, (6), pp. 523-540. (1994)

23. J. S. R. Jang, 'ANFIS: adaptive-network-based fuzzy inference system', IEEE Transactions on Systems, Man and Cybernetics, 23, (3), pp. 665-685. (1993)

24. J. S. R. Jang, and C. T. Sun, 'Neuro-fuzzy modeling and control', Proceedings of the IEEE, 83, (3), pp. 378-406. (1995)

25. J. S. R. Jang, and C. T. Sun, and E. Mizutani, Neurofuzzy and soft computing: a computational approach to learning and machine intelligence. Prentice Hall. (1997) 